\title{
Laser doppler myography (LDMi): A novel non-contact measurement method for the muscle activity
}

\author{
L Scalise $^{1}, \mathrm{~S} \mathrm{Casaccia}^{1}, \mathrm{P}_{\text {Marchionni }}{ }^{1}, \mathrm{I}_{\text {Ercoli }}{ }^{1}$, EP Tomasini $^{1}$ \\ 1: Dipartimento di Ingegneria Industriale e Scienze Matematiche (DIISM) \\ Università Politecnica delle Marche
}

\begin{abstract}
Background and aims: Electromyography (EMG) is considered the gold-standard for the evaluation of muscle activity. Transversal and dimensional changes of the muscle, during muscle activity, generate vibrational phenomena which can be measured by Laser Doppler Vibrometry (LDVi). There is a relationship between muscle contraction and vibrational activity, therefore, some information on fundamental muscle parameters can be assessed without contact with LDVi. In this paper, we explore the possibility to relate the EMG signal causing the muscle contraction and the vibrational activity also measureable on the muscle. A novel non-contact measurement method Laser Doppler myography (LDMi) - aiming to measure the vibrational behavior of muscle during contraction, is presented herein. Correlations with some parameters normally measured with EMG are reported.

Materials and methods: The proposed method has been compared with standard superficial EMG (sEMG). Signals produced with sEMG and laser Doppler myography have been simultaneously acquired and processed to test correlations on a population of 20 healthy volunteers. Tests have been carried out on the flexor carpi ulnaris and the tibialis anterior muscles (left and right).

Results: Results show that it is possible to measure: The timing of muscle activation (max differences: $440 \mathrm{~ms}$ ), the amplitude of the signals acquired during activation respect to the signals during rest $(\mathrm{S} / \mathrm{N})$, the correlation between the $\mathrm{S} / \mathrm{N}$ of the $\mathrm{sEMG}$ and LDMi signals at different levels of force ( $P>0.89)$, and to assess muscle fatigue.

Conclusions: LDMi is a valid measurement technique for the assessment of muscle activity and fatigue. It is a non-contact method and this characteristic could suggest its use together with low level laser therapy pre-, intra- and post-LLLT sessions to evaluate the efficacy and effects of the treatments without the need for invasive electrodes.
\end{abstract}

Key words: Electromyography $\cdot$ Laser Doppler Vibrometry $\cdot$ muscle activation time

\section{Introduction}

Neurology, orthopedics, sport medicine and rehabilitation therapy are only some of the many medical fields where muscle activity and muscle performances are studied. The gold standard instrumentation used for the evaluation of muscles is the Electromyography (EMG) 1). EMG aims to measure muscle activity through the assessment of electrical activity generated

Addressee for Correspondence:

Dr. Lorenzo Scalise

Dipartimento di Ingegneria Industriale e Scienze

Matematiche (DIISM)

Università Politecnica delle Marche 60131 Ancona (ITALY)

tel: +39-071-220-4442 fax: +39-071-220-4813

email: 1.scalise@univpm.it during contraction. EMG has been widely used for many decades, its use is proven and widely accepted by clinicians and the scientific community and it is considered the gold standard method for the measurement of muscle activity. EMG is operated directly on the muscle fibers by the use of needle electrodes which are in direct contact with the muscle fibers, but most commonly this approach is substituted by the use of superficial electrodes applied on the skin, corresponding to the muscle or part of the muscle that is under observation; in this case we have named the technique superficial electromyography (sEMG) 1,2 ). Both of these approaches have to be considered as

Received Date: February 8th, 2013

Accepted Date: November 14th, 2013 
contact and invasive measurement methods, also the sEMG method requires the direct application of electrodes onto the subject's skin, even if sEMG can be considered less invasive in respect to EMG which uses needle electrodes.

Therefore, even if sEMG is widely accepted, a limiting factor could be removed if the muscle activity is assessed without the need of applying any transducer, electrode or cable to the skin surface. In fact, with no physical links (cables) between the instrumentation and the patient it would be possible to operate the measurement with no or a very limited collaboration with the patient; and also the complexity and the time duration of the exam (skin preparation, electrodes placement, cable positioning, etc.) would be reduced.

This paper is focused on the presentation of a novel non-contact measurement method to detect muscle activity. The proposed method is based on the observation that the muscle activity and in particular the muscle contraction generates a variety of signal types: electric, mechanical, acoustic, and so on. In particular, the electric activity generated during muscle activation can be detected by EMG (or sEMG), while it is observed that a vibrational signal is also detectable due to the contraction of the longitudinal muscle fibers. This phenomenon was already observed by Rohrbaugh 3), and it is called mechanical myogragraphy. Previous experience using accelerometers to detect such vibrational behavior were limited by the invasive effect of the transducers and by the difficulties due to the fixation of the transducer mass to the skin surface ${ }^{3)}$. A very promising approach has been recently proposed by Rohrbaugh ${ }^{3}$ ) based on the use of a Laser Doppler Vibrometry (LDVi) which is able to detect muscle vibrations. LDVi is a laser interferometric measurement instrument which allows very precise vibration measurements from distance and without contact with the measurement. In recent years, it has been widely used for biomedical applications and it has been demonstrated to be a valid instrument for the assessment of the vibrational behavior of the human tissues ${ }^{3-8)}$.

The aim of this paper is therefore to present a non-contact measurement method based on LDVi for the detection of muscle activity, named Laser Doppler Myography (LDMi). The reported method has been tested on a population of 20 healthy subjects to monitor the mechanical activity of the flexor carpi ulnaris and the tibialis anterior muscles. Moreover we also present preliminary results correlating the LDMi-based method to the sEMG and to the force strength generated during the test. The potential for LDMi to be used for assessment of a patient's condition pre- and post low level laser therapy (LLLT) for muscle damage or other problems can also be considered.

\section{Materials and methods}

The study is focused on the detection of the muscle contraction from two muscles located on the arm and leg of humans: the flexor carpi ulnaris and the tibialis anterior muscles, respectively. The test involved the simultaneous acquisition of the sEMG signal and the LDMi signal during an isometric contraction of these muscles.

In order to record the sEMG signal it is necessary to apply two Ag-AgCl electrodes on the skin in correspondence to the right and left flexor carpi ulnaris/tibialis anterior while a reference electrode is positioned on the wrist for the flexor carpi ulnaris (bipolar configuration, Figure 1) and on the ankle for the tibialis anterior (bipolar configuration, Figure 2). Before the

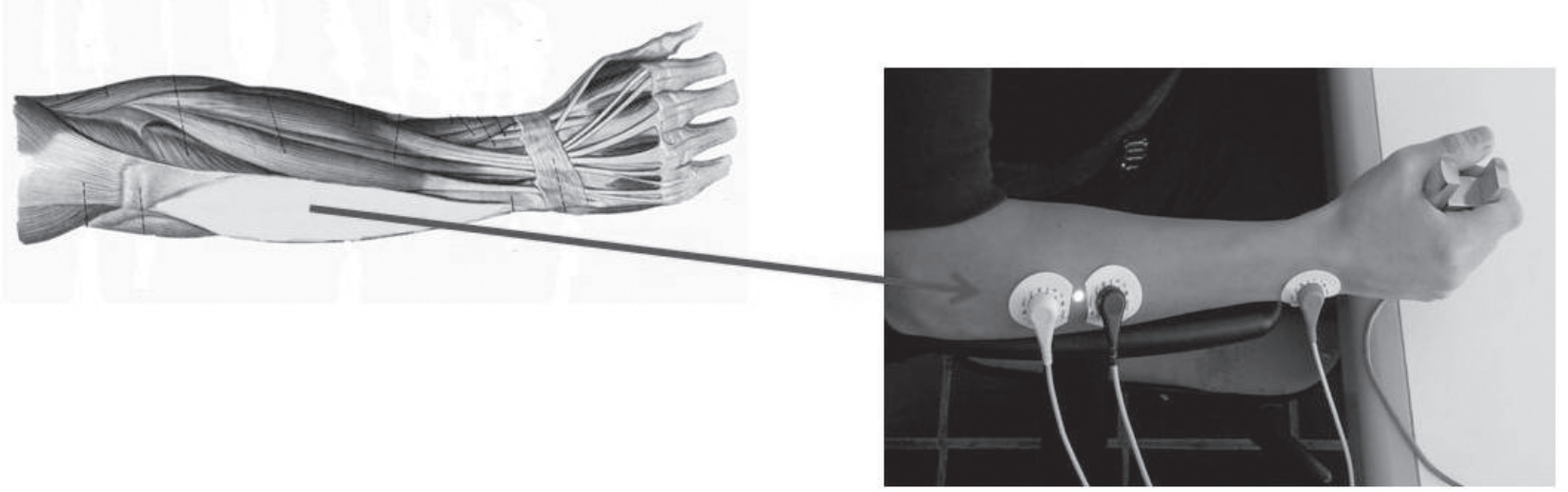

Figure 1. Representation of the flexor carpi ulnaris muscle (left); sEMG electrodes and LDMi laser spot positions during test. 
application of the electrodes, the skin was shaved and cleaned and cables were fixed in order to limit as much as possible the effect of noise. The correct positioning of patient's arm or leg under observation is a fundamental aspect of the measurement method. During the test on the arm muscle, the subject was asked to sit on a chair with the elbow flexed at a right angle and the dorsal side of the forearm in a horizontal downwards position and for the muscle contraction he/she must contract the hand holding an object (Figure 1). Attention must be paid to the fact that the muscle bicep brachii was not activated during the test and the contraction was isometric. For the analysis of the tibialis anterior muscle, the subject was asked to isometrically contract the tibialis anterior muscle, bringing the tip of the foot upwards (Figure 2).

The LDMi signal is operated by a single point instrumentation for the measurement of the velocity of rigid surfaces (PDV100; calibration accuracy \pm 0.05 $\mathrm{mm} / \mathrm{s}$, bandwidth $0.05 \mathrm{~Hz}-22 \mathrm{kHz}$, spot dimension $<1$ mm, working wavelength He-Ne $632.8 \mathrm{~nm}$ ). The velocity of the vibration of the target (in $\mathrm{mm} / \mathrm{s}$ ) was measured. For this, the laser beam was aimed perpendicularly to the skin surface of the arm and of the leg at a distance of about $30 \mathrm{~cm}$ and the laser spot was aimed between the skin electrodes (Figure 1).

sEMG and LDMi signals were acquired by a 12 bit acquisition board (ML865 PowerLab 4/25T). The surface EMG signal was filtered by a bandpass filter on the band 10-200 Hz and by a Notch filter to reduce the $50 \mathrm{~Hz}$ noise (filter band: $49.5-50.5 \mathrm{~Hz}$ ). Both the acquired signals were sampled at a frequency of 1 $\mathrm{kHz}$, synchronously. A sketch of the experimental setup realized for the data collection is reported in

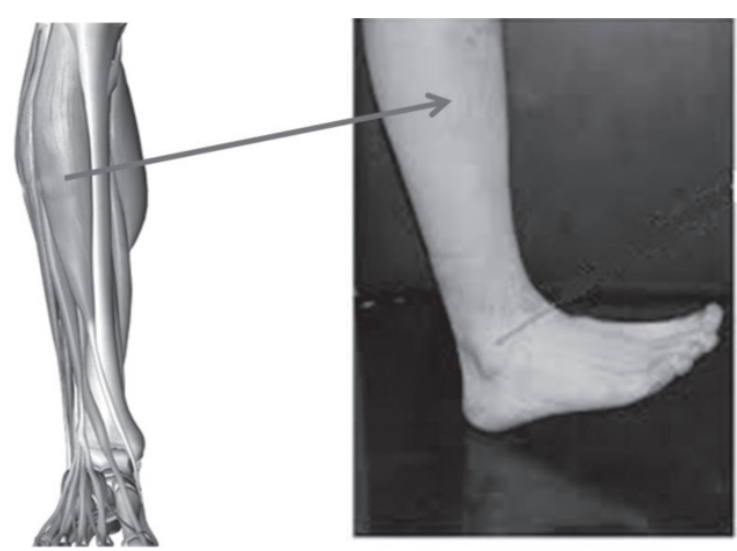

Figure 2. The isometrical contraction operated during tests for the case of the tibialis anterior.

\section{Figure 3}

The study population comprised 20 healthy subjects (10 females and 10 males) of mean weight $65 \pm 10$ $\mathrm{kg}$, height $170 \pm 10 \mathrm{~cm}$, and $24 \pm 5$ years. The measurement protocol operated for the tests was characterized by an initial period of $3 \mathrm{~s}$ of rest, followed by a $10 \mathrm{~s}$ of muscle activation and concluded by a period of $3 \mathrm{~s}$ of rest after the muscle contraction. Each of the subjects contributing to the study repeated the test 4 times. The data set had a total length of $16 \mathrm{~s}$. For each test the data set was characterized by 2 signals: the surface EMG signal and the LDV signal. Both the left and the right arms were measured.

The timings of muscle contraction for each of the 2 measurement methods utilized were calculated. A dedicated algorithm in Matlab ${ }^{\mathrm{TM}}$, specifically developed for this process, was developed with the scope to automatically calculate the timing of the activation. The algorithm calculates the root mean squared (RMS) of the acquired signal during the contraction phase and during muscle resting. The muscle activation is calculated on each signal as the first crossing of a threshold value; the threshold was calculated as 3 times the RMS value of the signal at rest. Muscle deactivation is determined as the second crossing of the threshold value.

A second set of tests was carried out in order to analyze the relationship between surface EMG and LDMi amplitudes. To this aim, the subjects were required isometrically to contract the isometrically muscle isometrically with 2 different force levels. Also in this case, sEMG and the LDMi signals were acquired and a contraction of $10 \mathrm{~s}$ was performed by the subject. Before and after each contraction, the muscle under observation was kept at rest to allow recovery to

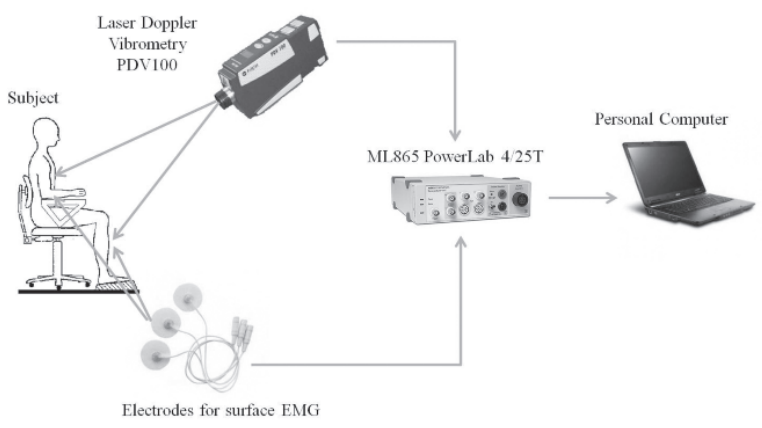

FIGURE 3. The experimental setup used for the tests 
the normal state. For this second series of tests, 3 subjects were selected with ages ranging between 22 to 25 and all of them were right-handed, healthy and not sport professionals. Each subject repeated 4 time the tes 4 times for each level of force at maximum and minimum level.

Finally in order to investigate if the LDMi method was able to determine the muscle contraction, we calculated the RMS (root mean square) of both the signals measured, as indicators of the signal sensitivity and of the muscle fatigue. For the test of the muscle fatigue, the subject under examination had to contract the right and left flexor carpi ulnaris at maximum force for $60 \mathrm{~s}$.

\section{Results}

Data collected from the test carried out were analyzed to determine:

- The exact timing of muscle activation in terms of the instant in time at which the contraction takes place (activation), time instant in time at which the muscle is de-contracted (deactivation), and the duration of the contraction (time of activation);

- The ratio of the amplitude of the signal acquired during activation in respect to the signals during rest $(\mathrm{S} / \mathrm{N})$ for both the measurement methods (sEMG and LDMi);

- S/N of the sEMG and LDMi signals at different levels of intensity of the muscle contraction;

- The effect of the muscle fatigue in terms of reduction of the signal amplitude.

Timing of muscle activity. In Figure 4, an example of the synchronously acquired signals is reported; the signals were measured during a test on the flexor carpi ulnaris: activation time (t1) and deactivation time (t2), calculated according the procedure previously presented, are reported for both the sEMG and LDMi signals.
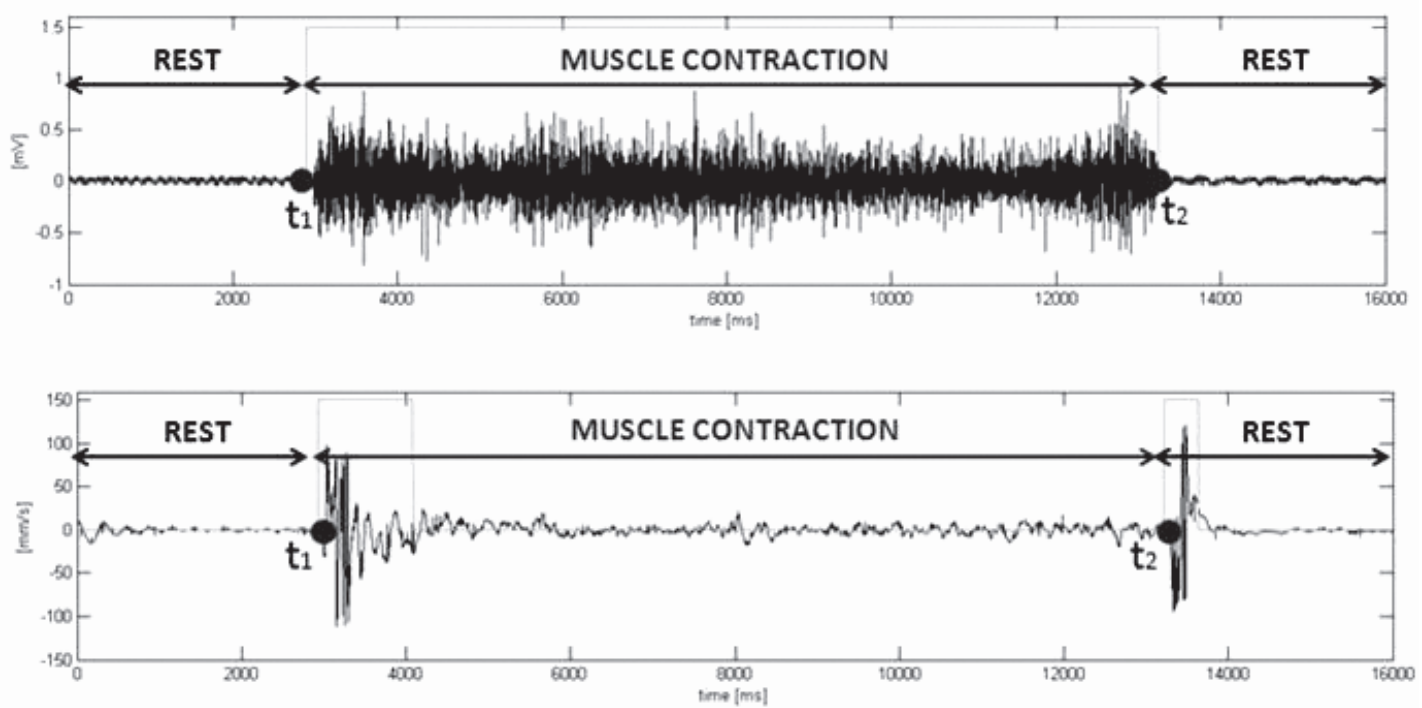

Figure 4. Timing of the muscle contraction: $s E M G$ signal and LDMi signal ( $\mathrm{t} 2-\mathrm{t} 1=$ total muscle contraction).

Table 4: Mean and standard deviation of the duration ( $\mathrm{t} 2-\mathrm{t} 1)$, activation ( $\mathrm{t} 1)$ and deactivation time ( $\mathrm{t} 2)$ for sEMG and LDMi (right and left flexor carpi ulnaris and right and left tibialis anterior)

\begin{tabular}{|c|c|c|c|}
\hline & \multicolumn{3}{|c|}{ Deviation between sEMG and LDMi values } \\
\hline & $\mathrm{t} 2-\mathrm{t} 1$ & $\mathrm{t} 1$ & $\mathrm{t} 2$ \\
\hline Right flexor carpi ulnaris $($ mean $\pm S D)$ & $0.12 \pm 0.09$ & $0.01 \pm 0.05$ & $0.13 \pm 0.09$ \\
\hline Left flexor carpi ulnaris $($ mean $\pm S D)$ & $0.23 \pm 0.44$ & $-0.008 \pm 0.04$ & $0.22 \pm 0.06$ \\
\hline Right tibialis anterior $($ mean $\pm S D)$ & $0.18 \pm 0.13$ & $-0.07 \pm 0.04$ & $0.12 \pm 0.11$ \\
\hline Left tibialis anterior $($ mean $\pm S D)$ & $0.15 \pm 0.12$ & $-0.05 \pm 0.03$ & $0.10 \pm 0.09$ \\
\hline
\end{tabular}


The surface EMG signal (Figure 4, top) showed a rapid increase of the amplitude when the subject was contracting the muscle of interest while the signal amplitude rapidly decreased when the contraction stopped and the subject relaxed. The LDMi signal, simultaneously acquired (Figure $\mathbf{4}$, bottom), is characterized by a first group of peaks in correspondence to the initial contraction (muscle activation, t1); a similar group of peaks is recorded again at the muscle deactivation ( $\mathrm{t} 2$ ). The time window between activation and deactivation was also calculated for both the EMG signals and LDMi signals.

In Table 1, the differences, in terms of mean and standard deviation, between the timing measured by sEMG and LDMi and the activation and deactivation events are reported for right/left flexor carpi ulnaris and right/left tibialis anterior.

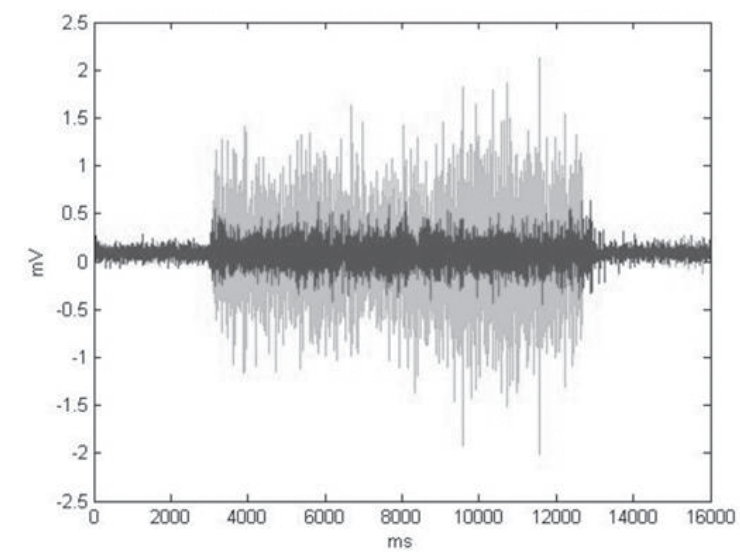

Figure 5. Scatter plot of sEMG $\mathrm{S} / \mathrm{N}$ and LDMi $\mathrm{S} / \mathrm{N}$ values for the right and left flexor carpi ulnaris (Pearson's coefficient: 0.95).

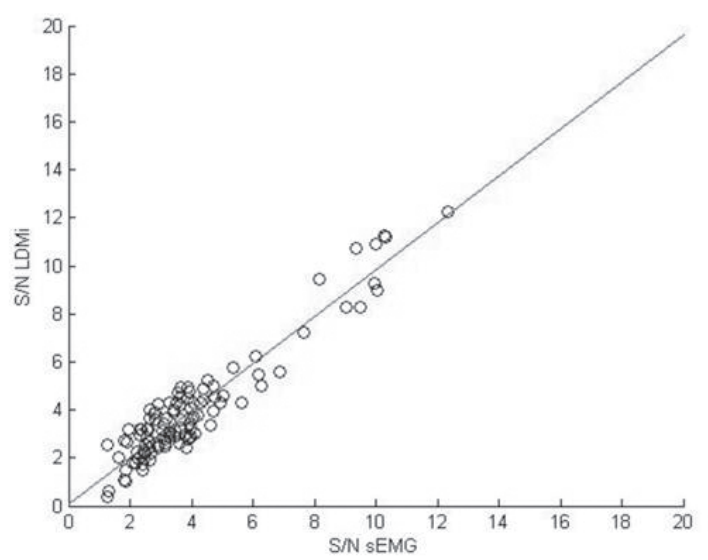

Figure 6. Scatter plot of sEMG S/N and LDMi S/N values for the right and left tibialis anterior (Pearson's coefficient: 0.89).
Amplitude of the signals. The RMS values of the signals for the rest condition $(\mathrm{t}<\mathrm{t} 1)$ and during contraction $(\mathrm{t} 1<\mathrm{t}<\mathrm{t} 2)$ were measured in order to evaluate methods sensitivities. In particular, this parameterwas calculated by the use of the signal-to-noise ratio $(\mathrm{S} / \mathrm{N})$ for the sEMG signal and the LDMi signal. The $\mathrm{S} / \mathrm{N}$ has been calculated as:

$$
S / N=\frac{\sqrt{\frac{1}{t_{2}-t_{1}} \sum_{i=t_{1}}^{t_{2}} \text { musclecontraction }_{i}{ }^{2}}}{\sqrt{\frac{1}{t_{1}} \sum_{j=0}^{t_{1}} \text { rest }_{j}{ }^{2}}}
$$

Where musclecontractioni is the $\mathrm{i}$-th values of the signal measured during contraction and restj is the $\mathrm{j}$-th value of signal measured during ret. The LDMi signal was filtered in order to remove noise; a wavelet filter was applied using a Sym 4 mother for the case of the flexor carpi ulnaris, while the Sym2 was used for the case of the tibialis anterior. Correlation between the $\mathrm{S} / \mathrm{N}$ values of the LDMi signal and the $\mathrm{S} / \mathrm{N}$ values of the sEMG signal - calculated using eq. 1 - are reported in the scatter plots depicted in Figures 5-7.

Relationship between sEMG and LDMi signals. To this aim, we calculated the $\mathrm{S} / \mathrm{N}$ of the signal measured by the 2 measurement methods (sEMG and LDMi) during the activation and resting of the muscles. Three subjects were selected for this test and each subject repeated the test 4 times for two levels of force: the maximum contraction of the muscle and a minimum

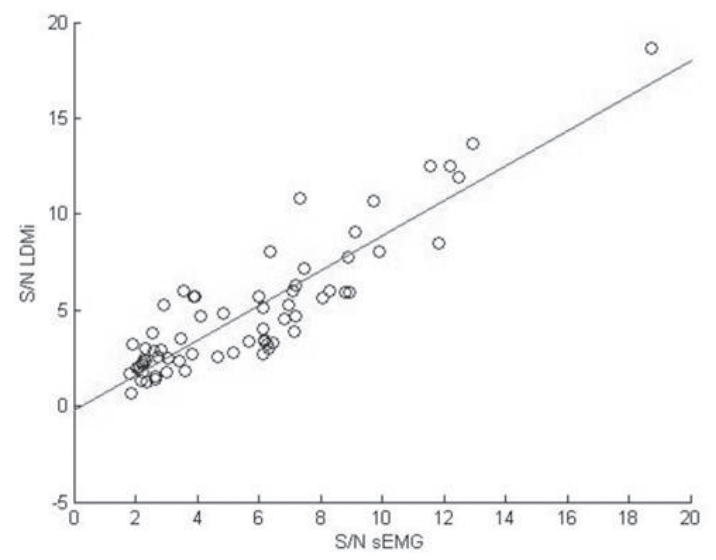

Figure 7. Scatter plot of sEMG S/N and LDMi S/N values for the right and left flexor carpi ulnaris and tibialis anterior (Pearson's coefficient: 0.93). 
level of force (subjectively determined). In Figure 8, the $\mathrm{S} / \mathrm{N}$ values of the LDMi and sEMG signals of the right/left flexor carpi ulnaris are reported in a scatter plot.

Muscle fatigue. The RMS value of the time signals and the slope of the interpolating line are known parameters for the evaluation of the muscle fatigue. The RMS value, according eq.1, is calculated during the muscle contraction for sEMG and LDMi signals. For this test, the acquisition time stands for 60s. Two examples of time series of the RMS of the sEMG and LDMi signals are reported in Figures 9 and 10, respectively.

\section{Discussion}

The use of LDMi for the measurement of the activation and deactivation events has been proved by the experiments described in the previous paragraph and reported in Table 1. In particular, it is possible to observe that the activation time ( $\mathrm{t} 1$ ) of the muscle observed is determined with minor differences in respect to the standard sEMG method; the max difference on the determination of the activation time was found for the case of the right tibialis anterior $(70 \pm 4 \mathrm{~ms})$. The determination of the deactivation time (t2) is less accurate in respect to the $\mathrm{t} 1$ for all the muscles group observed (Table 1); in particular larger differences were reported for the left flexor carpi ulnaris (220 $\pm 6 \mathrm{~ms}$ ). It is anyway worthy of observation that the deactivation time also for the case of the sEMG traces had a less stepwise shape in respect to the activation time (Figure 4), this causing a larger variance in the determination of the instant of deactivation.

It is also interesting to observe the good correlation between the $\mathrm{S} / \mathrm{N}$ values of the LDMi and sEMG signals; Pearson's coefficient values ranged between 0.88 and 0.95 , as reported in Figures 5-7. Such a strong correlation could suggest that the amplitude of the sEMG signal from a muscle unit could be derived from the signal generated by the LDMi

From Figure 8, it is possible to observe that in tandem with the increase in force, there is an increase of the sEMG signal amplitude as well as of the LDMi signal. We compared the various signals and calculated the Pearson coefficient to be 0.88 .

Finally, it is possible to observe a negative slope of the interpolating lines for both the measurement methods used (sEMG and LDMi) leading to the possibility of detecting the effect of muscle fatigue in terms of the value of the angular coefficient (slope) of the interpolating line (Figure 10), in a similar manner as

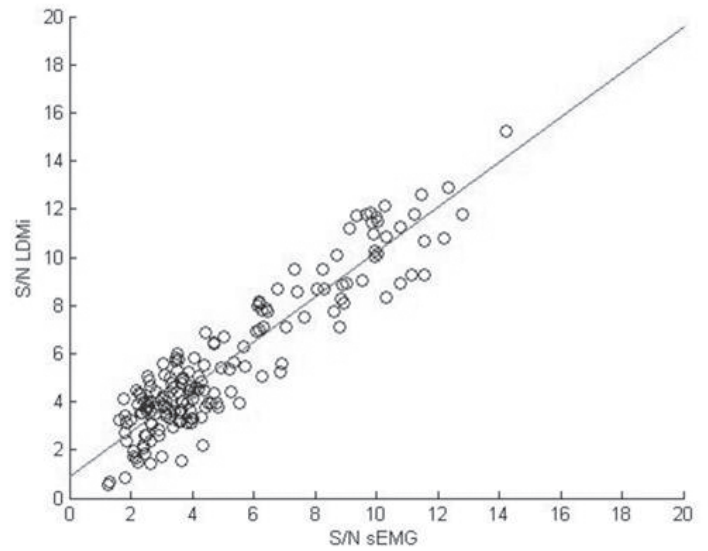

Figure 8. Scatter plot of $s E M G ~ S / N$ and LDMi $\mathrm{S} / \mathrm{N}$ values for isometric contraction of the right and left flexor carpi ulnaris muscle (Pearson's coefficient: 0.88).

for the sEMG signal (Figure 9).

\section{Conclusions}

The purpose of this study was to report on the preliminary results obtained with a non-contact measurement method (Laser Doppler Myography, LDMi) for the evaluation of muscle activity and to investigate the correlation between the surface EMG signal and LDMi signals. The analysis of the correlation between the gold standard technique and the proposed measurement method was carried out in a population of 20 subject and the muscles studied were the right and left flexor carpi ulnaris and the right and left tibialis anterior. We focused our study on the determination of activation/deactivation time instants and the results show that the detection timing is sufficiently accurate (a maximum standard deviation of $440 \mathrm{~ms}$ was reported). Our conclusion is therefore that it was possible to survey the flexor carpi ulnaris and tibialis anterior activation time and duration of activation with LDVi signal with a minimal difference of time duration on the contraction time of $<50$ and $230 \mathrm{~ms}$, respectively.

A second purpose of this study was to determine the correlation between the sEMG and the LDMi signals. The high values of the Pearson coefficients reported in the results paragraph testify to the possibility to relate the amplitudes of these signals; as a consequence it is possible to assert that, as far as the determination of the signal amplitude was concerned, the LDMi represented a non contact alternative. It is also important to underline the importance of signal filter- 


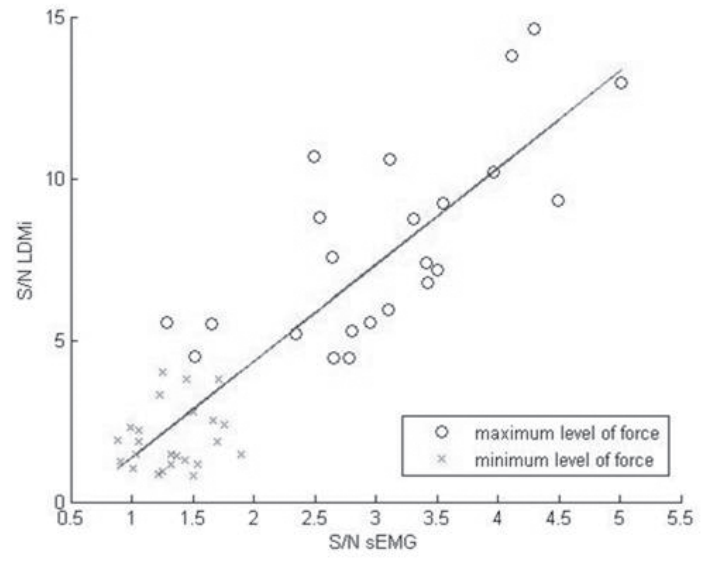

Figure 9. RMS of the sEMG signal during activation.

ing; for the sEMG signal filtering was set at a standard value and was operated during the acquisition, whereas for LDMi signal, filtering was operated after acquisition by wavelet processing. The reported correlation between the sEMG signals and LDMi signals was calculated with Pearson's Coefficient to be 0.93 (the mean over all tests). A similar correlation was also reported in the case of minimal and maximal muscle contraction. Moreover, with RMS parameters it was possible to evaluate the muscle fatigue. For the case of a $60 \mathrm{~s}$ contraction on the right and left flexor carpi ulnaris the muscle fatigue were detected by means of the negative slope of regression line.

A potential future application of the proposed measurement method could explore the possibility to integrate this method with low level laser therapy (LLLT) on muscles 9-12). Finally the possibility of the

\section{References}

1: Webster JG: Medical instrumentation: application and design ( $3^{\text {rd }}$ ed.). 1998, John Wiley \& Sons New York.

2: Medved V and Cifrek M. Kinesiological Electromyography. In (Klika V, ed.) Biomechanics in Applications, 2012, InTech (Available from: http://www.intechopen.com/books/biomechanicsin-applications/kinesiological-electromyography).

3: Rohrbaugh JW, Sirevaag EJ and Richter EJ (2012). Laser Doppler Vibrometry measurement of the mechanical myogram. Proc. of the $10^{\text {th }}$ International Conference on Vibration Measurements by Laser and Noncontact Techniques. AIP, 1457:266-274.

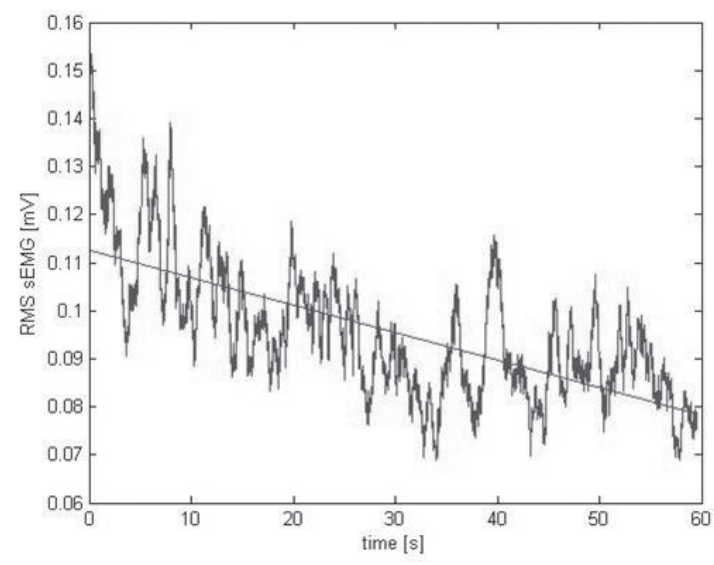

Figure 10. RMS of the LDMi signal during activation.

use of LDMi as an evaluating procedure looks particularly stimulating, providing quantitative and qualitative indicators for the results of physiotherapeutic and rehabilitative procedures including LLLT. The non-contact nature of the proposed method in particular suggest the possibility the laser-based rehabilitative treatments (i.e. LLLT) to be carried out in combination with LDMi as a valid support concomitantly with the therapeutic procedures either in the pre-, intra- and post-treatment phases. The use of LLLT and LDMi would give an immediate feedback to the clinician of the muscle status in relation to the specific LLT treatment selected, allowing an optimization of such treatment; this aspect being a fundamental paradigm of optimal therapy treatment for the cases of: stroke, brain injury, degenerative central nervous system, temporary palsy, sport injuries or spinal cord trauma 13-16).

4: De Melis M, Morbiducci U, Scalise L, Tomasini EP, Delbeke D, Baets R, Van Bortel LM, Segers P (2008). A non contact approach for the evaluation of large artery stiffness: a preliminary study. American Journal of Hypertension, 21:1280-1283.

5: Scalise L and Morbiducci U (2008). Non contact cardiac monitoring from carotid artery using optical vibrocardiography. Medical Engineering $\mathcal{E}$ Physics, 30(4):490-497.

6: Scalise L, Marchionni P and Ercoli I (2010). A noncontact optical procedure for precise measurement of respiration rate and flow, Proc. SPIE 7715 , Biophotonics: Photonic Solutions for Better Health 
Care II, 77150G.

7: Scalise L, Rossetti F and Paone N (2007). Hand vibration: non-contact measurement of local transmissibility. International Archieves of Occupational and Environmental Health 81(1):31-40.

8: Castellini P, Scalise L and Revel GM (2000). Vibration measurements for diagnosis of structural defects on human teeth. Measurement, 27(1):29-42.

9: Tullberg M, Alstergren PJ, Ernberg MM. (2003) Effects of low-power laser exposure on masseter muscle pain and microcirculation, Pain 105, 89-96.

10: Asagai Y, Ueno R, Miura Y, Ohshiro T (1995): Application of low reactive-level laser therapy (LLLT) in patients with cerebral palsy of the adult tension athetosis type. Laser Therapy, 7: 113-118.

11: Ohshiro T, and Calderhead RG (1988). Low Level Laser Therapy: A Practical Introduction. John Wiley \& Sons, Chichester, UK.

12: Yoshimi A, Watanabe Y, T Oshiro, Yamamoto K (2005). Suppression of myotonia in cerebral palsy and adjunctive effect of low level laser therapy on intensive functional training. Laser Therapy, 4:171178.

13: Hashmi JT, Huang YY, Osmani BZ, Sharma SK, Naeser MA, Hamblin MR (2010). Role of low-level laser therapy in neurorehabilitation. $P M E R$, 12(2):S292-305.

14: Okuni I, Harada T, Ushigome N, Ohshiro T, Musya Y, Maruyama Y, Suguro T, Tsuchiya K (2008). Low Level Laser Therapy(LLLT) for Facial Palsy Patients. Laser Therapy 17(3):135-139.

15: Morimoto Y, Saito A, Tokuhashi Y (2013). Low level laser therapy for sports injuries. Laser Therapy, 22(1): 17-20.

16: Ushigome N, Harada T, Okuni I, Ohshiro T, Musya Y, Mizutani K, Maruyama Y, Suguro T, Tsuchiya K (2008). Effects of Low Level Laser Therapy (LLLT) On Spasticity Caused By Cerebral Vascular Accidents (CVAS). Laser Therapy, Vol. 17, No. 2, p.95-99.

The contents of this article were originally presented as an oral paper at Laser Florence 2012 on November $10^{\text {th }} 2012$ Florence, Italy 